\title{
ANDROGEN PRODUCING ADENOMA IN A PATIENT WITH NON-DIAGNOSED CONGENITAL ADRENAL HYPERPLASIA.
}

\author{
Authors: Kosák M¹, Hana jr. V1', Michalsky D², Kubinyi J³ , Hana V ${ }^{1}$
}

1 - Third Department of Medicine, General University Hospital and First Faculty of Medicine, Charles University, Prague, Czech Republic; 2 - First Department of Surgery, General University Hospital and First Faculty of Medicine, Charles University, Prague, Czech Republic; Institute of Nuclear Medicine, General University Hospital and First Faculty of Medicine, Charles University,

Prague, Czech Republic

\section{INTRODUCTION}

Androgen secreting adrenal tumor is a rare cause of androgen excess in women. There are only few case-reports of androgen producing adenoma in a patient with congenital adrenal hyperplasia.
Our patient, 61 year old woman was referred to our department for progressive hirsutism and androgenic alopecia. She didn't mention neither excessive facial hair growth nor balding until age of 59 when rapidly progressive balding and overt hirsutism started. She has had 3 childbirths and 3 abortions in the past.

\section{RESULTS}

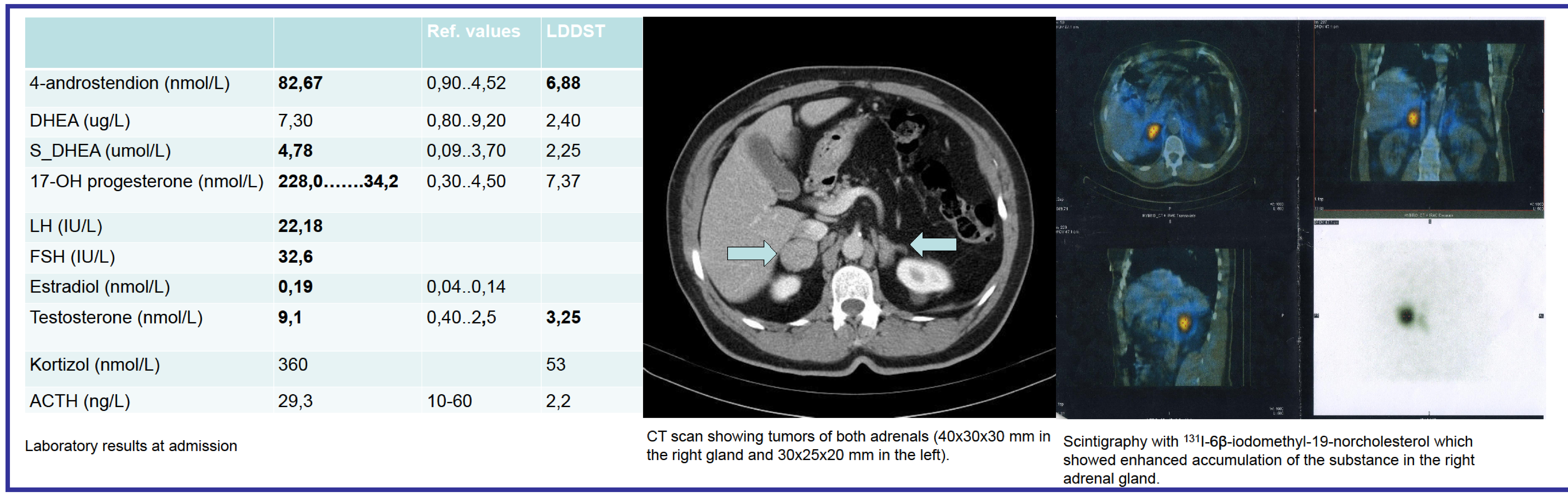

\section{TREATMENT}

With suspicion on androgen producing adenoma laparoscopic adrenalectomy of the right adrenal was performed:

\begin{tabular}{ll} 
& \\
\hline 4-androstendion (nmol/L) & $\mathbf{8 , 0 6}$ \\
DHEA (ug/L) & 2,40 \\
S_DHEA (umol/L) & 1,03 \\
17-OH progesterone (nmol/L) & $\mathbf{1 2 9 , 2 6}$ \\
LH (IU/L) & 27,53 \\
FSH (IU/L) & 47,15 \\
Estradiol (nmol/L) & $\mathbf{2 9 4 , 4}$ \\
Testosterone (nmol/L) & 1,86 \\
& \\
ACTH (ng/L) & $\mathbf{2 9 4 , 4}$
\end{tabular}

Laboratory results after surgery
Histology revalead yellowish tumor in the adrenal cortex $40 \times 30 \times 20 \mathrm{~mm}$. cortical adenoma adenoma. Surrounding cortex with hypeplasia:

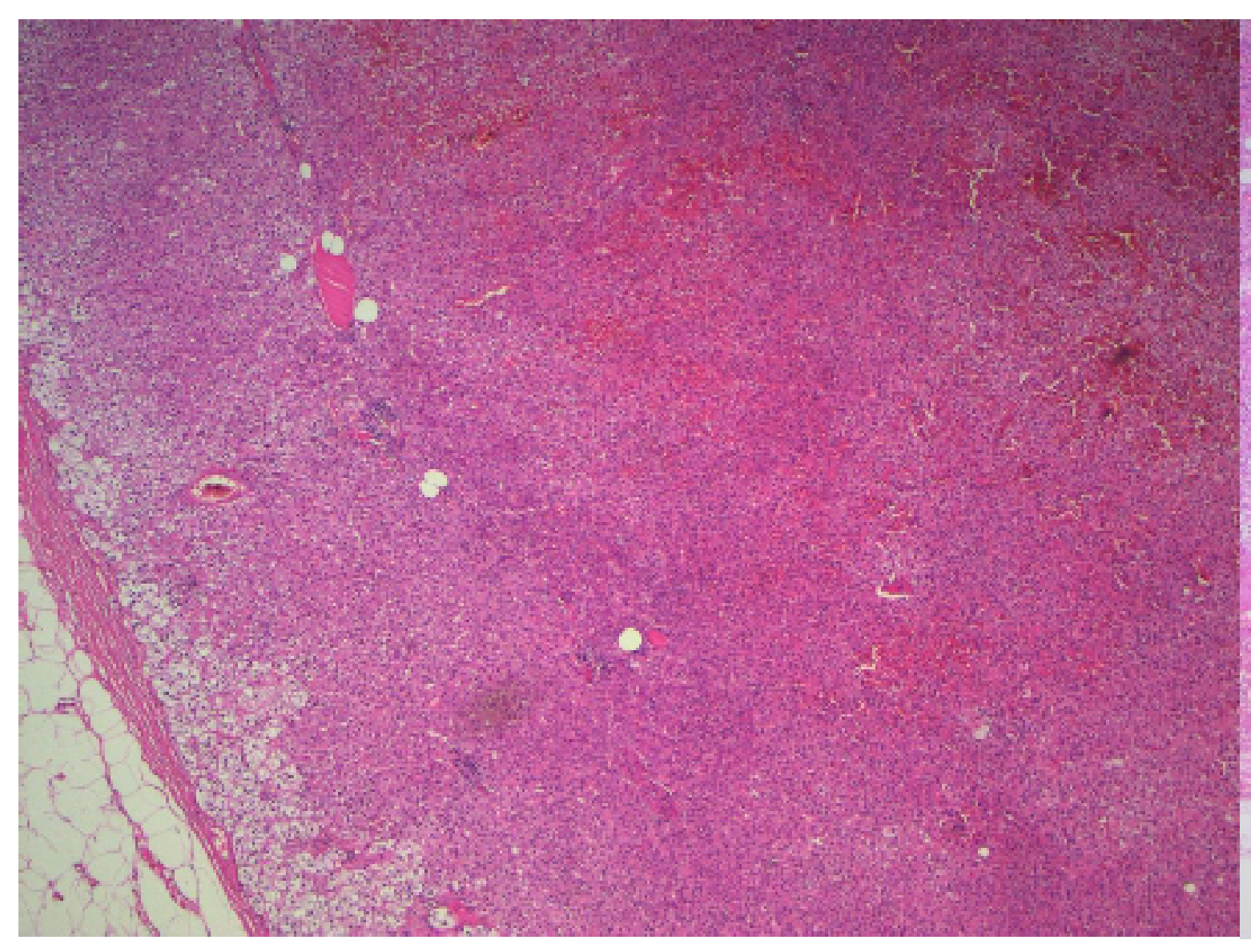

\section{Genetic test}

Due to peristent elevation of 17-OHP, despite normalization of androgens (testosterone and 4-androstendion) signficantly elevated before surgery, test for suspected congenital adrenal hyperplasia (21 OH def.) was permormed. Our patient is compound heterozygote for $\mathrm{CAH}$.

Treatment with corticosteroids, to supress the androgen overproduction from the left adrenal was commenced.

\section{CONCLUSIONS}

Androgen secreting adenoma is a rare cause of hyperandrogenism. In the presented case adenoma had developed in a patient with, so far undiagnosed, congenital adrenal hyperplasia. 